\title{
Biocarbón de bambú como mejorador de la fertilidad del suelo en caña de azúcar
} Bamboo biocharcoal as soil fertility enhancer in sugar cane

\author{
Gabriela Orozco Gutiérrez ${ }^{1}$, Laura Medina Telez ${ }^{2 *}$, Antonio Elvira Espinosa² \\ y Jeovani Francisco Cervantes Preciado ${ }^{1}$
}

\begin{abstract}
The deterioration exhibited by the soils is largely due to the scarcity and poor quality of water, and to the decrease in their fertility, in particular. Biocharcoal or biochar is a byproduct of the pyrolysis of residual biomass, which helps to recover soil fertility. The present work aims to evaluate organic bamboo biochar amendments in Saccharum spp. var. CP 72-2086. For this purpose, a factorial design $2^{2}$, $\mathrm{T0}=$ control without biochar (S-AB); $\mathrm{T}^{1}=10 \mathrm{t} \mathrm{ha}{ }^{-1}(\mathrm{BBV})$ and $\mathrm{T2}$ $=20 \mathrm{t} \mathrm{ha}^{-1}$ (BBV) of Bambusa vulgaris biochar; T3 = $10 \mathrm{t} \mathrm{ha}^{-1}$ (BBVV) and T4 = $20 \mathrm{t} \mathrm{ha}^{-1}$ (BBVV) of Bambusa vulgaris vittata biochar, distributed in random blocks. In the statistical model, the assessed variables were root biomass, height, diameter and number of shoots. The response variables were analyzed by means of an analysis of variance (ANOVA) based on a general linear model and the comparison of Tukey means with a significance level of $5 \%$. The application of bamboo biochar in the cultivation of sugarcane improved the physical and chemical properties of the soil by increasing the field capacity, the permanent wilting point, the moisture content, $\mathrm{pH}$ and the cation exchange capacity. Bamboo biochar in a concentration of $20 \mathrm{t} \mathrm{ha}^{-1}$ is a good organic amendment to the soil since it increases the availability of nutrients benefiting the growth of this species.
\end{abstract}

Key words: Nutrient bioavailability, growth, amendment, enhancer, pyrolysis, soil.

\section{Resumen}

El deterioro de los suelos se debe, en gran medida, a la escasez y mala calidad del agua, y en particular a la disminución de la fertilidad de los mismos. El biocarbón es un subproducto de la pirolisis de biomasa residual que ayuda a recuperar la fertilidad edáfica. El presente trabajo tiene como objetivo evaluar enmiendas orgánicas de biocarbón de Bambusa vulgaris en Saccharum spp. var. CP 72-2086. Para ello, se utilizó un diseño factorial $2^{2}$, T0 = testigo sin biocarbón (S-AB); T1=10 t ha-1 (BBV) y T2= $20 \mathrm{t} \mathrm{ha}^{-1}$ (BBV) de biocarbón de Bambusa vulgaris; T3= $10 \mathrm{t} \mathrm{ha}^{-1}$ (BBVV) y T4=20 t ha-1 (BBVV) de biocarbón de Bambusa vulgaris vittata, distribuidos en bloques al azar. En el modelo estadístico las variables evaluadas fueron: biomasa de raíz, altura, diámetro y número de brotes. Las variables respuesta se analizaron por medio de un análisis de varianza (ANOVA) con base en un modelo lineal generalizado, y la comparación de medias de Tukey con un nivel de significancia de $5 \%$. La aplicación de biocarbón de bambú en el cultivo de caña de azúcar mejoró las propiedades físicas y químicas del suelo, ya que aumentó la capacidad de campo, el punto de marchitez permanente, la humedad, el pH y la capacidad de intercambio catiónico. El biocarbón de bambú en una concentración de 20 t ha-1 es una buena enmienda orgánica al suelo, puesto que incrementa la disponibilidad de nutrimentos lo que beneficia el crecimiento del cultivo.

Palabras clave: Biodisponibilidad de nutrimentos, crecimiento, enmienda, mejorador, pirolisis, suelo.

Fecha de recepción/Reception date: 27 de mayo de 2020

Fecha de aceptación/Acceptance date: 23 de julio de 2020

${ }^{1}$ Instituto Nacional de Investigaciones Forestales, Agrícolas y Pecuarias. Centro de Investigación Regional Pacífico Centro. Campo Experimental Tecomán. México.

2Instituto Tecnológico Superior de Ciudad Serdán. Ciudad Serdán, Puebla, México.

*Autor por correspondencia; correo-e: arual1410zelet@gmail.com 


\section{Introducción}

El deterioro que presentan los suelos se debe, en gran medida, a la escasez y mala calidad del agua, y en particular a la disminución de la fertilidad de los mismos. Esta última ocurre por una seria condición de erosión, degradación, contaminación y pérdida de la materia orgánica que inciden de manera directa en la producción alimentaria (Semarnat-Colpos, 2003). En consecuencia, la nutrición de los cultivos es afectada por deficiencias nutrimentales graves; por lo que, en algunos casos, se incurre en excesos en la fertilización. Lo anterior afecta, directamente, la calidad de los alimentos y la salud ambiental, además del incremento de los costos de producción.

Existe preocupación por el uso indiscriminado de fertilizantes químicos, ya que por lo general las dosis empleadas no son las requeridas para los cultivos y ocasionalmente no se considera el aporte natural de nutrimentos que puede hacer el suelo. Si la oferta nutrimental no satisface la demanda de un cultivo, no se alcanzan los rendimientos máximos que permite la peculiaridad de cada agroecosistema, y como efecto colateral, se genera una degradación del suelo (Escalante, 2013).

Ante la preocupante situación del deterioro del suelo, es imperioso implementar estrategias para mejorar su calidad. Adicionalmente, el cambio climático tiende a agravar la situación, por su efecto en la disponibilidad de agua (deficiencia o exceso), sequía extrema o las lluvias torrenciales, altas o bajas temperaturas que afectan la producción de alimentos y la conservación de la calidad del suelo. Por ello, el llamado es a llevar a cabo una gestión de los suelos que asegure tanto su conservación, como la producción (Funes-Monzote y Monzote, 2001; Sánchez et al., 2011).

Los suelos de México exhiben una fuerte degradación física, química y biológica. Existen tecnologías para contrarrestarlas, las cuales ayudan a recuperar su fertilidad y secuestrar carbono, como la aplicación del biocarbón, producto obtenido mediante el tratamiento térmico de la pirolisis de biomasa, bajo un entorno limitado o sin oxígeno (Lehmann et al., 2006). La pirolisis es el proceso termoquímico que se efectúa a temperaturas que varían entre 300 y $600{ }^{\circ} \mathrm{C}$. 
El biocarbón está constituido, principalmente, por carbono (70-80\%). Este material se considera un componente importante en las propuestas para mitigar las emisiones de gases de efecto invernadero como $\mathrm{CO}_{2}$ y NO $\mathrm{N}_{2}$, debido a que los retiene y captura (Lehmann et al., 2006). También, es relevante como mejorador de las características y propiedades físicas, químicas y biológicas del suelo (Lehmann y Joseph, 2009). Se ha documentado que el biocarbón en el suelo puede aumentar la capacidad de intercambio catiónico y favorecer el desarrollo de microrganismos (Liang et al., 2006); asimismo, propicia la retención de nutrimentos y la estructura del suelo (Liu et al., 2012).

El biocarbón se elabora de diferentes materias primas, las más usadas son madera de árboles, residuos agroindustriales, residuos agrícolas, estiércol de ganado y ave, además de residuo de podas (Pérez, 2015). Aunque su producción y aplicación al suelo es una tecnología empleada en varias partes del mundo (Lehman y Joseph, 2009; Steiner, 2010; Waters et al., 2011), en México hay poca información disponible sobre el particular. En general se recomienda que el biocarbón se produzca solo donde hay suficiente biomasa disponible, tal es el caso de los subproductos de actividades agroforestales o residuos orgánicos urbanos, que pueden ser usados como materia prima.

El bambú de la especie Bambusa vulgaris Schrad. ex J.C. Wendl. está ampliamente distribuida en el territorio nacional; sin embargo, es uno de los bambúes más subutilizados debido a que, en comparación con otras especies, no es estructural. $B$ vulgaris se utiliza en otros países en la industria del papel (Banik, 2015; Banik, 2016). Además, el bambú constituye una alternativa sustentable para sustituir a la madera, ya que requiere, únicamente, 5 años para ser aprovechado, a diferencia de los 20 a 40 años que se necesitan para los recursos forestales maderables. Durante el proceso de aprovechamiento de los culmos (tallos) de bambú dentro de una plantación forestal comercial, se generan residuos sólidos, los cuales pueden usarse para elaborar biocarbón.

En este trabajo se presentan los estudios que se realizaron en el desempeño de dos biocarbones producidos a partir de culmos sobremaduros de bambú (Bambusa vulgaris Schrad. ex J.C. Wendl. var. vulgaris y Bambusa vulgaris Schrad. ex J.C. 
Wendl. var. vittata), y la evaluación de los mismos en la fertilidad del suelo en el cultivo de caña de azúcar variedad CP 72-2086.

\section{Materiales y Métodos}

Se implementó un ensayo en un vivero perteneciente al Campo Experimental Tecomán, del Instituto Nacional de Investigaciones Forestales Agrícolas y Pecuarias (INIFAP) localizado en la costa del Pacífico mexicano, en el municipio Tecomán del estado de Colima; se ubica entre los $18^{\circ} 57^{\prime} 58.9^{\prime \prime}$ a $18^{\circ} 57^{\prime} 58.8^{\prime \prime} \mathrm{N}$ y los $103^{\circ} 50^{\prime} 33^{\prime \prime}$ a $103^{\circ} 50$ '32.6" O; altitud de $59 \mathrm{~m}$, donde predomina el clima semiseco muy cálido $\left[\mathrm{BS}_{1}\left(\mathrm{~h}^{\prime}\right)\right]$. La temperatura media anual es de $30^{\circ} \mathrm{C}$, con una precipitación media anual de $810.6 \mathrm{~mm}$, con régimen de lluvias, principalmente, en verano (INEGI, 2017).

Para el ensayo, se usaron plantas de caña de azúcar de la variedad comercial CP 722086 , procedente de cultivo in vitro que fueron establecidas en invernadero durante un período de 2 meses (en etapa de amacollamiento o pelillo), en tubetes de polietileno de $190 \mathrm{~cm}^{3}$. Las plantas se trasplantaron a raíz desnuda en bolsas de polietileno negro, de alta densidad, de $12 \times 23 \mathrm{~cm}(3 \mathrm{~L})$ y suplementadas con $2 \mathrm{~kg}$ de tierra agrícola, la cual previamente se analizó químicamente.

\section{Muestreo y análisis químico del suelo}

Se utilizó suelo agrícola procedente del Ingenio Quesería S.A. de C.V. (Zona cañera) para el trasplante de las plantas en bolsas de polietileno. El muestreo se llevó a cabo tomando completamente al azar seis de las bolsas con suelo para obtener tres muestras homogéneas de $1 \mathrm{~kg}$ cada una. Este procedimiento se siguió al inicio (suelo sin enmienda) y al final del ensayo (suelo enmendado con biocarbón a los 70 días).

Se realizaron determinaciones de las propiedades físicas y químicas bajo la NOM.021RECNAT-2002 (Semarnat, 2002) con sus correspondientes metodologías: pH (AS02); contenido de humedad (AS-05); contenido de materia orgánica (AS-07); 
nitrógeno total (AS-08); textura (AS-09); fósforo (AS-11); capacidad de intercambio catiónico y bases intercambiables (AS-12); micronutrimentos (AS-14); conductividad eléctrica (AS-18); Ca Mg, Na y P (AS-19); carbonatos totales (AS-20).

\section{Biocarbón de bambú y análisis realizados}

Se utilizaron culmos de bambú sobremaduro (muerto) de 25 años de edad, correspondientes a Bambusa vulgaris var. vulgaris Schrad. ex Wendl. y Bambusa vulgaris var. vittata Schrad. ex Wendl., provenientes de la parcela "La máquina" del Ingenio Quesería S.A. de C.V., las cuales se seleccionaron bajo un muestreo al azar con un intervalo de humedad en campo de 20-25\%. Los culmos extraídos se aserraron en trozas de $20 \mathrm{~cm}$ y se pirolizaron en un horno vertical NOVATECH $^{\circledR}$ durante $4.5 \mathrm{~h}$, a una temperatura promedio de $550{ }^{\circ} \mathrm{C}$. Una vez carbonizados, el tamaño de partícula se redujo utilizando un molino extractor $K R E T O R^{\circledR}$, y se determinó el porcentaje de partículas retenidas en los tamices: 6.3, 4.0, 2.0, 0.84, $0.5,0.4,0.074,0.044 \mathrm{~mm}$ en un agitador vibratorio MOMBOLDY ${ }^{\circledR}$.

Los análisis físicos y químicos de los biocarbones se realizaron bajo las siguientes normas: pH ASTM D 1293-18 (ATM, 1999), materia orgánica UNE-EN-14774 (UNEEN, 2010); nitrógeno, fósforo y potasio según la NOM-021-RECNAT-2000 establecidas en las metodologías AS-08; AS-10 y AS-13, respectivamente (Semarnat, 2002).

\section{Diseño experimental}

Se aplicó un arreglo factorial $2^{2}$, cuyos factores fueron BBV (Bambusa vulgaris), BBVV (Bambusa vulgaris vittata) y los niveles: 10 y $20 \mathrm{t} \mathrm{ha}^{-1}$, más un testigo; los tratamientos evaluados fueron: T0 (S-AB) sin aplicación de biocarbón; $\mathrm{T} 1=10 \mathrm{t} \mathrm{ha}{ }^{-1}$ de BBV; T2= $20 \mathrm{tha}^{-1}$ de BBV; T3=10 tha-1 de BBVV y T4=20 $\mathrm{t} \mathrm{ha-1}$ de BBVV. La unidad experimental fue cada planta de caña. Se utilizó un diseño experimental de bloques al azar con seis repeticiones, con un total de 30 unidades experimentales de Saccharum spp. var. CP 72-2086. El factor de bloqueo fue el tamaño de partícula. 


\section{Aplicación del biocarbón}

Hernández-Hernández (2015) usó dosis de residuos de carbón vegetal correspondientes a $0,10,15,20,25,30,35$ y $40 \mathrm{t} \mathrm{ha}^{-1}$ con resultados favorables en la aplicación de 10 y $20 \mathrm{t} \mathrm{ha}^{-1}$; es por ello, que se utilizaron estas en el experimento que se documenta. Previo a la aplicación, ambos biocarbones se pesaron de manera individual para cada unidad experimental, para obtener las dosis equivalentes a $10 \mathrm{y}$ $20 \mathrm{t} \mathrm{ha}^{-1}$, que se adicionaron en la parte superior de cada bolsa; posteriormente, fueron regadas. La duración de la experimentación fue de 70 días, después de la aplicación del biocarbón.

\section{Variables respuesta evaluadas en el cultivo}

Las variables estudiadas fueron volumen de raíz, altura, diámetro de cada uno de los brotes primarios y secundarios de las plantas de caña, así como el número de brotes totales. Se realizaron evaluaciones cada 15 días durante 70 días después del trasplante; en cada medición se seleccionaron al azar seis individuos por tratamiento para su evaluación.

\section{Análisis estadístico}

Las variables respuesta se analizaron mediante un análisis de varianza (ANOVA) con base en un modelo lineal generalizado y la comparación de medias de Tukey, con $5 \%$ de nivel de significancia, con el paquete estadístico Minitab versión 17. El modelo estadístico que se utilizo fue el propuesto por Sitún (2005):

$$
Y_{i j}=\mu+T_{i}+B_{j}+\varepsilon_{i j}
$$


Donde:

$\mu=$ Media general de la variable de respuesta por evaluar

$T_{i}=$ Efecto del $i$-ésimo nivel de los diferentes tratamientos a utilizar

$B_{j}=$ Efecto del $j$-ésimo bloque o repetición

$\varepsilon_{i j}=$ Error experimental asociado a la $i$-j-ésima unidad experimental

\section{Resultados y Discusión}

\section{Caracterización del biocarbón de bambú}

Los resultados de los análisis se muestran en el Cuadro 1. Los biocarbones de ambas especies presentaron un pH moderadamente ácido, en contraste con otros estudios en los que se han caracterizado como materiales alcalinos y que se han utilizado como mejoradores del balance del pH edáfico (Amonette y Joseph, 2009). El pH alcalino del biocarbón se debe a la relación de la temperatura de producción y el tipo de materia prima; es decir, el biocarbón a base de madera tiende a tener un $\mathrm{pH}$ más alto que el biocarbón hecho de residuos de cosecha y estiércol (Gul et al., 2015; Abujabhah et al., 2016). 
Cuadro 1. Análisis de macro y micronutrimentos en el suelo y análisis de biocarbón de Bambusa vulgaris Schrad. ex J.C. Wendl. y Bambusa vulgaris Schrad. ex J.C. Wendl. var. vittata.

\begin{tabular}{cccccc}
\hline Parámetro & Unidad & Suelo (día 0) & Suelo (día 70) & BBV & BBVV \\
\hline $\mathrm{CE}$ & $\mathrm{dS} \mathrm{m^{-1 }}$ & 0.76 & 1.56 & 2.35 & 1.290 \\
$\mathrm{CIC}$ & $\mathrm{meq} 100 \mathrm{~g}^{-1}$ & 12.85 & 24.69 & - & - \\
$\mathrm{MO}$ & $\%$ & 6.09 & 5.91 & 86.94 & 92.28 \\
$\mathrm{P}$ & $\mathrm{ppm}$ & 20 & 64.08 & 8532.8 & 6836.1 \\
$\mathrm{~K}$ & $\mathrm{ppm}$ & 259.99 & 377.83 & 312.6 & 458.4 \\
$\mathrm{Ca}$ & $\mathrm{ppm}$ & 2005.54 & 4283.14 & 2122.5 & 631.5 \\
$\mathrm{Mg}$ & $\mathrm{ppm}$ & 262.83 & 259.07 & 1350 & 1201.5 \\
$\mathrm{Na}$ & $\mathrm{ppm}$ & 2.83 & 50.83 & 33 & 34.48 \\
$\mathrm{Cu}$ & $\mathrm{ppm}$ & 1.7 & 1.35 & 12.93 & 12.02 \\
$\mathrm{Fe}$ & $\mathrm{ppm}$ & 35.08 & 26.9 & 57.31 & 42.6 \\
$\mathrm{Zn}$ & $\mathrm{ppm}$ & 3.22 & 2.31 & 16.96 & 27.39 \\
$\mathrm{~B}$ & $\mathrm{ppm}$ & 0.421 & 0.32 & 17.11 & 50.84 \\
\hline
\end{tabular}

$\mathrm{BBV}=$ Bambusa vulgaris; $\mathrm{BBVV}=$ Bambusa vulgaris vittata; $\mathrm{CE}=$ Conductividad eléctrica; $\mathrm{CIC}=$ Capacidad de intercambio catiónico; $\mathrm{MO}=$ Materia orgánica; $\mathrm{P}=$ Fósforo, $\mathrm{K}=$ Potasio; $\mathrm{Ca}=$ Calcio; $\mathrm{Mg}=$ Magnesio; $\mathrm{Na}=$ Sodio; $\mathrm{Cu}=$ Cobre; $\mathrm{Fe}=$ Hierro; $\mathrm{Zn}=$ Zinc; $\mathrm{B}=$ Boro.

En investigaciones de caracterización del biocarbón se indica que el intervalo del pH de la mayoría de los biocarbones varía entre 6.2 y 13 (Chan y Xu, 2009; Srinivasarao 
et al., 2013). En el presente ensayo el pH de los diferentes biocarbones de bambú fueron similares al de la madera de roble (6.38) y al de la palma de Acai (6.3) (Lehmann et al., 2011; Nunes et al., 2019). Ambas especies tienen componentes como celulosa y hemicelulosa, los cuales son comunes en todas las plantas leñosas y en algunas especies de bambú, tal es el caso del género Bambusa. Por lo tanto, la utilización de biocarbón a partir de Bambusa vulgaris y Bambusa vulgaris vittata son alternativas que alcalinizan el $\mathrm{pH}$ del suelo.

De acuerdo a Jia et al. (2013), el pH del biocarbón se relaciona con la cantidad de ácidos orgánicos y fenólicos derivados de la descomposición térmica de la celulosa y la hemicelulosa. De manera similar, Gul et al. (2015) señalan que una razón para el aumento del $\mathrm{pH}$ en el suelo con biocarbón es la presencia en su superficie de grupos carboxilo e hidroxilo, cargados negativamente que unen iones $\mathrm{H}^{+}$de la solución del suelo, lo que reduce la concentración de iones $\mathrm{H}^{+}$en la solución del suelo y aumenta su $\mathrm{pH}$.

La conductividad eléctrica de los biocarbones fue de $2.35 \mathrm{dS} \mathrm{m}^{-1}$ en BBV y $1.290 \mathrm{dS} \mathrm{m}^{-1}$ en BBVV; es decir, moderadamente salinos (Brady y Weil, 2008); por lo que, de acuerdo a Hernández-Hernández (2015) no existe riesgo de que ocurran problemas de salinidad para el cultivo de la caña de azúcar, cuando se utilicen los biocarbones como enmendantes. Los resultados de la presente investigación coinciden con lo citado por Concilco et al. (2018), quienes consignan que la CE del biocarbón fue de $2.55 \mathrm{dS} \mathrm{m}^{-1}$ en Bambusa vulgaris var. vulgaris y Bambusa oldhammi Munro.

El contenido de carbono total en el BBVV fue de $53.55 \%$, el cual resultó mayor al de BBV (50.54\%); sin embargo, fueron más altos que el de un biocarbón de cocoite (33\%) (Hernández-Godínez, 2015). Aunque, inferiores al carbón total (84.2 \%) de un biocarbón de $B$. vulgaris vittata y al de $B$. vulgaris ( $86.3 \%$ ), ambos a una temperatura de $650^{\circ} \mathrm{C}$ (Brito y Filho, 1987). Cabe destacar que, el contenido de carbono está muy relacionado con la temperatura de pirolización (Demirbas, 2004; Uchimiya et al., 2010).

El contenido de fósforo fue de $8532.8 \mathrm{mg} \mathrm{kg}^{-1}$ para el BBV, el cual fue superior al de BBVV (6 $836.1 \mathrm{mg} \mathrm{kg}^{-1}$ ); resultado excelente para el biocarbón de bambú. Ambos 
valores superan los $743 \mathrm{mg} \mathrm{kg}$-1 de un biocarbón de bagazo de caña (HernándezGodínez, 2015). Castellanos et al. (2000); Jia et al. (2013) y Yao et al. (2013) proponen que los biocarbones con alta concentración de fósforo pueden emplearse como fertilizantes de liberación lenta, pero resaltan la necesidad de establecer correctamente la dosis, frecuencia y el tiempo de aplicación; ya que el fósforo es de liberación lenta (Hernández-Hernández, 2015).

El contenido de fósforo en el suelo aumentó, considerablemente, debido a la liberación por parte de los biocarbones utilizados. Yao et al. (2013) sugiere aplicar el biocarbón de forma directa al suelo, como fertilizante, para la producción sostenible de cultivos y como secuestrador de carbono.

El contenido de materia orgánica fue mayor en el BBVV, con $92.28 \%$ comparado a $86.44 \%$ en el BBV. Ambos resultados son superiores a los indicados por Domínguez (2013) para un biocarbón usado como sustrato orgánico en el crecimiento de plántulas de caña de azúcar, en el que se obtuvo 60.50 y $63.50 \%$ de materia orgánica, y a los de Concilco et al., (2018) en un biocarbón comercial de B. vulgaris y B. oldhammi, el cual presentó $0.25 \%$.

En el Cuadro 1 se presenta la concentración total de Mg: 1 350, $1201 \mathrm{mg} \mathrm{kg}^{-1}$; Ca: 2 122.5, $631.1 \mathrm{mg} \mathrm{kg}^{-1}$; $\mathrm{Na}: 33,34.48 \mathrm{mg} \mathrm{kg}^{-1}$; Cu: 12.93, $12.02 \mathrm{mg} \mathrm{kg}^{-1}$; Fe: 57.31, $42.6 \mathrm{mg} \mathrm{kg}{ }^{-1}$; Zn: 16.96, 27.39; y B: $17.11,50.84 \mathrm{mg} \mathrm{kg}^{-1}$ de BBV y BBVV. Los resultados de ambos biocarbones en estos elementos fueron mayores a los citados por Rojas (2017) en un biocarbón de cascarilla de café, y a los de Concilco et al. (2018) para un biocarbón comercial de B. vulgaris y B. oldhamii. 


\section{Efecto del biocarbón en el suelo}

De acuerdo a los resultados del análisis de suelo antes y después de la aplicación del biocarbón, el pH aumentó de 6.93 a 7.69, por lo que se clasifica como moderadamente alcalino. Acorde a otros estudios, el biocarbón con pH alcalino puede utilizarse en suelos ácidos como material de encalado para el mejoramiento del pH (Rojas, 2017), ya que este se relaciona con la disponibilidad nutrimental para el crecimiento y desarrollo de las plantas (Gul et al., 2015).

La conductividad eléctrica (CE) se elevó de 0.76 a $1.56 \mathrm{dS} \mathrm{m}^{-1}$ (Cuadro 1), la cual se considera moderadamente baja, lo cual indica que el suelo está libre de la presencia de sales, condición ideal para el crecimiento de la caña de azúcar. Además, la capacidad de intercambio catiónico (CIC) se incrementó de 12.85 a 24.69 meq $100 \mathrm{~g}^{-1}$. Hay estudios en los que se demuestra que el biocarbón puede alterar la fertilidad del suelo, por medio de un aporte directo de nutrimentos o bien por el aumento de la CIC (Liang et al., 2006); lo que favorece la retención de nutrimentos y evita las pérdidas por lixiviación (Liang et al., 2006).

El contenido de materia orgánica (MO) disminuyó de 6.09 a $5.91 \%$. Según los parámetros de la NOM-021-RECNAT-2000 (Semarnat, 2002) los suelos volcánicos son de contenido medio y los no volcánicos son muy altos en MO; por lo tanto, el suelo utilizado en el experimento que aquí se documenta es de contenido medio. La MO tiene un efecto importante en la CIC, debido a que esta potencialmente retiene e intercambia nutrimentos, además de promover la actividad microbiológica del suelo (Lehman y Joseph, 2009; Abujabhah et al., 2016). Con la aplicación del biocarbón de bambú hubo un incremento en el fósforo, potasio, calcio, magnesio y sodio, a su vez disminuyeron algunos micronutrimentos como el cobre, hierro, zinc y boro (Cuadro 1).

Las propiedades físicas del suelo antes y después de la aplicación de biocarbón se resumen en el Cuadro 2. La densidad aparente (Da) fue de $1.60 \mathrm{~g} \mathrm{~cm}^{-3}$; con base en la textura corresponde a un suelo franco arenoso (Semarnat, 2002). Asimismo, en las propiedades físicas del suelo hubo un cambio, ya que presentó una mayor retención de humedad en los tratamientos donde se aplicó biocarbón, la cual se favoreció por la capacidad de campo y el punto de marchitez permanente (Cuadro 2). 
Cuadro 2. Análisis de propiedades físicas del suelo antes y después de la aplicación de biocarbón

\begin{tabular}{lcc}
\hline \multicolumn{1}{c}{ Determinaciones } & $\begin{array}{c}\text { Antes de la aplicación de } \\
\text { biocarbón }\end{array}$ & $\begin{array}{c}\text { Después de la aplicación de } \\
\text { biocarbón }\end{array}$ \\
\hline Capacidad de campo \% & 40.00 & 42.50 \\
Punto de marchitez permanente \% & 21.31 & 22.00 \\
Humedad aprovechable \% & 19.19 & 20.00 \\
Densidad aparente $\mathrm{g} \mathrm{cm}^{3}$ & 1.60 & 1.60 \\
\hline
\end{tabular}

\section{Efecto del biocarbón en la altura de las plantas de caña de azúcar}

El análisis de varianza efectuado a los resultados de la variable altura (Cuadro 4), establece que la adición de biocarbón al suelo con los diferentes tratamientos, tuvieron un efecto significativo sobre la altura de las plantas. De acuerdo a la comparación de medias entre los tratamientos con la prueba de Tukey (Cuadro 3), indica que al aplicar (20 t ha-1 BBVV) tratamiento 4, se registró una mayor altura en las plantas de caña de azúcar significativamente superior a las del tratamiento 2 (BBV $20 \mathrm{t} \mathrm{ha}^{-1}$ ). En los tratamientos control (S-AB), 1 (BBV con $10 \mathrm{t} \mathrm{ha}^{-1}$ ), y 3 (BBVV con $10 \mathrm{t} \mathrm{ha}^{-1}$ ) no hubo diferencia estadística significativa. Lo anterior, indica que la variable altura de planta por sí sola no representa un criterio sólido para determinar un tratamiento superior en particular, sino que deben tomarse en consideración el resto de las variables agronómicas. 
Cuadro 3. Efecto del biocarbón en las variables de crecimiento en el cultivo de Caña de azúcar variedad CP 72-2086.

\begin{tabular}{lccccc}
\hline \multicolumn{1}{c}{$\begin{array}{c}\text { Variables de } \\
\text { crecimiento }\end{array}$} & \multicolumn{4}{c}{ Tratamientos } \\
\cline { 2 - 6 } & S-AB & BBV_10 & BBV_20 & BBVV_10 & BBVV_20 \\
\hline Altura $(\mathrm{cm})$ & $60.06 \pm 1.53 \mathrm{ab}$ & $62.90 \pm 1.53 \mathrm{ab}$ & $59.24 \pm 1.53 \mathrm{~b}$ & $63.66 \pm 1.53 \mathrm{ab}$ & $66.86 \pm 1.53 \mathrm{a}$ \\
Diámetro $(\mathrm{mm})$ & $6.53 \pm 0.18 \mathrm{a}$ & $6.74 \pm 0.18 \mathrm{a}$ & $6.17 \pm 0.18 \mathrm{a}$ & $6.89 \pm 0.18 \mathrm{a}$ & $6.91 \pm 0.18 \mathrm{a}$ \\
No. de brotes & $8.37 \pm 0.51 \mathrm{~b}$ & $11.00 \pm 0.51 \mathrm{ab}$ & $11.20 \pm 0.51 \mathrm{a}$ & $9.41 \pm 0.51 \mathrm{ab}$ & $11.62 \pm 0.51 \mathrm{a}$ \\
Volumen de raíz $\left(\mathrm{cm}^{3}\right)$ & $76.62 \pm 8.44 \mathrm{c}$ & $84.81 \pm 8.44 \mathrm{bc}$ & $110.91 \pm 8.44 \mathrm{ab}$ & $110.40 \pm 8.44 \mathrm{ab}$ & $117.00 \pm 8.44 \mathrm{a}$ \\
\hline
\end{tabular}

T0 $(S-A B)=$ Sin aplicación de biocarbón; T1 $=10 \mathrm{t} \mathrm{ha}^{-1}$ de BBV; T2 $=20 \mathrm{t} \mathrm{ha}^{-1} \mathrm{de}$ $\mathrm{BBV} ; \mathrm{T} 3=10 \mathrm{t} \mathrm{ha}^{-1}$ de BBVV; T4 $=20 \mathrm{t} \mathrm{ha}^{-1}$ de BBVV. Las medias que no comparten una letra son significativamente diferentes, prueba de Tukey $(P \leq 0.05)$.

Cuadro 4. Análisis de varianza de las variables respuesta.

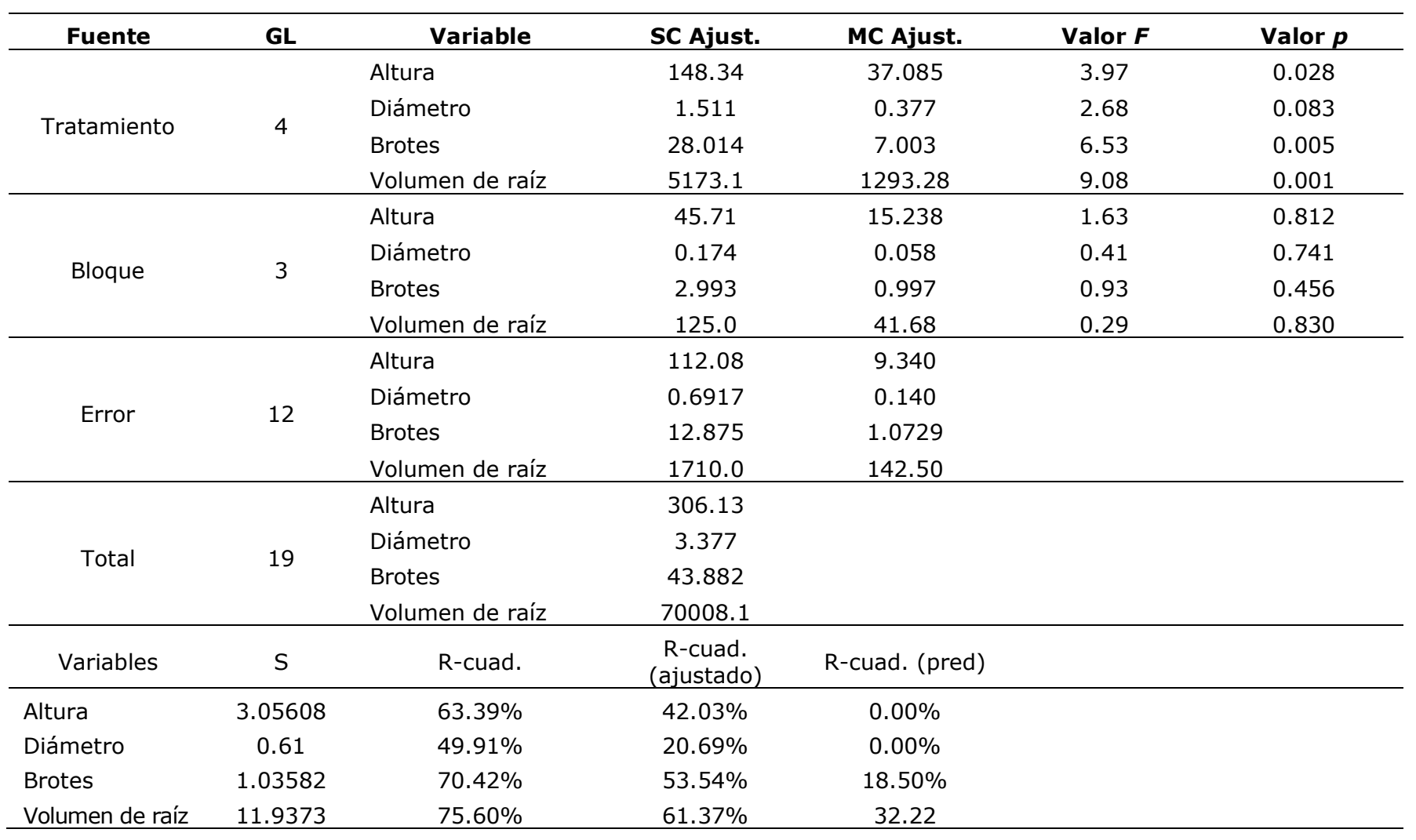

Tratamientos evaluados: T1 (BBV $10 \mathrm{t} \mathrm{ha}^{-1}$ ), T2 (BBV $20 \mathrm{t} \mathrm{ha}{ }^{-1}$ ), T3 (BBVV $10 \mathrm{t} \mathrm{ha}^{-1}$ ), T4 (BBVV $20 \mathrm{t} \mathrm{ha}^{-1}$ ), T5 (S/AB). 


\section{Efecto del biocarbón en el diámetro}

El análisis de varianza efectuado en la variable diámetro de tallo (Cuadro 4) y la comparación de medias de Tukey entre los tratamientos, establece que la adición de biocarbón al suelo con las dosis empleadas a los 70 días, no tuvieron un efecto significativo sobre esta variable, ya que el efecto atribuido por todos los tratamientos es igual. La variable diámetro de tallo durante el desarrollo o crecimiento del cultivo de la caña de azúcar, muestra incrementos pocos pronunciados, es decir, es una característica particular de este híbrido.

\section{Efecto del biocarbón en la población de número de tallos}

La variable número de tallos molederos por hectárea es una de las variables de mayor relevancia, ya que impacta directamente el potencial de rendimiento en campo. El análisis de varianza efectuado a los resultados de la variable número de tallos (Cuadro 4), establece que la adición de biocarbón al suelo con los diferentes tratamientos, tuvieron un efecto significativo sobre esta variable. De acuerdo a la comparación de medias entre los tratamientos con la prueba de Tukey (Cuadro 3), indica que al aplicar

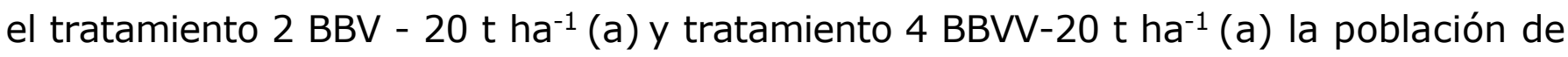
tallos es estadísticamente superior al tratamiento control (b). En los tratamientos 1 BBV-10 t ha-1 (ab), 3 BBVV-10 t ha-1 (ab) las medias fueron iguales.

\section{Efecto del biocarbón en el volumen de raíz}

La comparación de medias de los tratamientos con la prueba de Tukey (Cuadro 3) indica que al aplicar el tratamiento 4 (BBVV-20 t ha-1), se registró un volumen de raíz estadísticamente significativo con respecto al tratamiento control (S-AB).

El análisis de varianza efectuado en la variable volumen de raíz (Cuadro 4), establece que la adición de biocarbón al suelo en los diferentes tratamientos, tuvieron un efecto significativo sobre esta variable. 


\section{Conclusiones}

La aplicación de biocarbón tuvo un efecto positivo en la altura, número de brotes y volumen de raíz de las plantas de caña de azúcar. Una mayor dosis de biocarbón promovió una tasa de crecimiento más alta en dichas plantas, con el biocarbón de Bambusa vulgaris vittata en una concentración de 20 t ha-1. El biocarbón de Bambusa vulgaris en una concentración de $20 \mathrm{t} \mathrm{ha}^{-1}$ solo influye en el número de brotes. El uso de biocarbón de bambú como opción de enmienda orgánica mejora la fertilidad del suelo y el ciclo de nutrimentos, lo que incide favorablemente en el crecimiento de las plantas. En este estudio, el biocarbón de bambú mejora la mayoría de los rasgos de crecimiento de las plantas de caña de azúcar, como su altura, diámetro, número de brotes totales y volumen de raíz, ya que el biocarbón previene la lixiviación de los nutrimentos y aumenta la retención de agua debido a su alta superficie y naturaleza porosa; en consecuencia, los nutrimentos están disponibles para el consumo de las plantas. En general las propiedades físicas y químicas del suelo mejoran mediante la aplicación del biocarbón. Los resultados positivos del estudio sugieren un beneficio potencial para mejorar la fertilidad del suelo y el crecimiento de cultivos como la caña de azúcar y con ello contribuir al desarrollo de la agricultura y la economía en el país.

\section{Agradecimientos}

Se agradece el financiamiento del proyecto fiscal INIFAP: Viabilidad y factibilidad en la propagación, conservación, establecimiento y manejo sostenible de cinco especies nativas de bambú leñoso en la región Pacífico- Centro de México. También al Ingenio Quesería S.A. de C.V. Y finalmente, al personal operativo, así como al laboratorio de Suelos, Planta y Agua del INIFAP Campo Experimental de Tecomán. 


\section{Conflicto de interés}

Los autores declaran no tener conflictos de intereses.

\section{Contribuciones por autor}

Gabriela Orozco Gutiérrez: trabajo de campo, análisis, escritura y responsable del proyecto integral; Laura Medina Telez: trabajo de campo, análisis estadístico, trabajo de laboratorio y de campo, análisis de datos; Antonio Elvira Espinosa: apoyo en análisis edáficos; Jeovani Francisco Cervantes Preciado: análisis de crecimiento de caña.

\section{Referencias}

Abujabhah, I. S., S. A. Bound, R. Doyle and J. P. Bowman. 2016. Effects of biochar and compost amendments on soil physico-chemical properties and the total community within a temperate agricultural soil. Applied Soil Ecology (98): 243-253. Doi: $10.1016 /$ j.apsoil.2015.10.021.

Amonette, J. E. and S. Joseph. 2009. Characteristics of biochar: Micro-chemical properties. Chapter 3. Biochar for Environmental Management: Science and Technology. Earthscan, London, UK and Sterling, VA, USA. 52 p.

American Society for Testing and Materials (ASTM). 1999. ASTM D1293-18, Métodos de prueba estándar para pH de agua. ASTM International. West Conshohocken, PA, USA. www.astm.org (20 de noviembre de 2019).

Banik, R. 2015. Morphology and growth. In: Liese, W. and M. Köhl (eds.). Bamboo the plant and its uses. Tropical forestry. Springer. Gewerbestr, Switzerland. pp. 43-89. Doi: $10.1007 / 978-3-319-14133-6$. 
Banik, R. 2016. Bambusa. In: Banik, R. (ed.). Silviculture of South Asian Priority Bamboos. Tropical Forestry. Springer. Singapore, Singapore. pp. 21-115. Doi: 10.1007/978-981-10-0569-5_3

Brady, N. and R. Weil. 2008. The Nature and Properties of Soils. 14th Ed Pearson/Prentice-Hall. New Jersey, USA. 975 p.

Brito, J. O and T. M. Filho. 1987. Produção e caracterização do carvão vegetal de espécies e variedades de bambu. IPEF 36(1):13-17. https://www.ipef.br/publicacoes/scientia/nr36/cap02.pdf (25 de mayo de 2020).

Castellanos J., Z., J. X. Uvalle-Bueno y A. Aguilar S. 2000. Manual de Interpretación de Análisis de Suelos y Aguas. $2^{a}$ Edición. Instituto de Capacitación para la productividad Agrícola. San Miguel de Allende, Gto., México. 226 p.

Concilco, A., E., A. Moreno R., M. García C., H. M. Quiroga G. y O. Á. García. 2018. Influencia del biocarbón aplicado al suelo sobre atributos de rendimiento y calidad de avena forrajera. Revista Terra Latinoamericana 36(3):221-228.

Doi: $10.28940 /$ terra.v36i3.375.

Chan, K. Y. and Z. H. Xu. 2009. Biochar: nutrient properties and their enhancement. Biochar for environmental management. Science, technology and implementation (1):67-84.

http://www.css.cornell.edu/faculty/lehmann/publ/First\%20proof\%2013-01-09.pdf (18 de febrero de 2020).

Demirbas, A. 2004. Effects of temperature and particle size on bio-char yield from pyrolysis of agricultural residues. Journal of Analytical and Applied Pyrolysis 72(2): 243-248. Doi: 10.1016/j.jaap.2004.07.003. 
Domínguez G., M. I. 2013. Evaluación y caracterización de sustratos orgánicos para el crecimiento de plántulas de caña de azúcar. Tesis de maestría. Instituto de enseñanza e investigación en ciencias agrícolas. Colegio de Postgraduados. Heroica Cárdenas, Tab., México. 91 p.

Escalante R., M. A. 2013. Biocarbones (biochars), caracterización y efecto en la biomasa y nutrición de NPK en una gramínea. Tesis doctoral. Instituto de enseñanza e investigación en ciencias agrícolas. Colegio de Postgraduados. Montecillo, Texcoco, Edo. de Méx., México. 137 p.

Funes-Monzote, F. y M. Monzote. 2001. Unir en un todo coherente una opción sustentable y productiva. Experiencia cubana de integración ganadería-agricultura sobre bases agroecológicas. Boletín de ILEIA (16): 22-24. http://www.leisaal.org/web/index.php/statistics/volumen-16-numero-4/2339-unir-en-un-todocoherente-una-opcion-sustentable-y-productiva (18 de diciembre de 2019).

Gul, S., J. K. Whalen, B. W. Thomas, V. Sachdeva and H. Deng. 2015. Physicochemical properties and microbial responses in biochar-amended soils: Mechanisms and future directions. Agriculture, Ecosystems and Environment (206): 46-59. Doi: 10.1016/j.agee.2015.03.015.

Hernández-Godínez, M. I. 2015. Fito estabilización de elementos potencialmente tóxicos por Jatropha curcas L., Acaulospora sp. y biochar. Tesis de maestría. Instituto de enseñanza e investigación en ciencias agrícolas. Colegio de Postgraduados. Montecillo, Texcoco, Edo. de Méx., México. 110 p.

Hernández-Hernández, M. 2015. Carbón vegetal como mejorador de un acrisol cultivado con caña de azúcar. Tesis de maestría. Instituto de enseñanza e investigación en ciencias agrícolas. Colegio de postgraduados. Heroica Cárdenas, Tab., México. 61 p. 
Instituto Nacional de Estadística, Geografía e Informática (INEGI). 2017. Anuario Estadístico y Geográfico de Colima. Colima, Col. México. 35 p.

Jia, M., F. Wang, Y. Bian, X. Jin, Y. Song, F. O. Kengara, R. Xu and X. Jiang. 2013. Effects of $\mathrm{pH}$ and metal ions on oxytetracycline sorption to maize-straw-derived biochar. Bioresource Technology (136): 87-93. Doi: 10.1016/j.biortech.2013.02.098.

Lehmann, J., J. Gaunt and M. Rondon. 2006. Bio-char sequestration in terrestrial ecosystems - A review. Mitigation and Adaptation Strategies for Global Change 11(2): 403-427. Doi: 10.1007/s11027-005-9006-5.

Lehmann, J. and S. Joseph. 2009. Biochar for environmental management. Science and technology. Earthscan. Second edition. UK-USA. 405 p. https://biocharinternational.org/book/ (10 de diciembre de 2019).

Lehmann, J., M. C. Rilling, J. Thies, C. A. Masiello, W. C. Hockaday and D. Crowley. 2011. Biochar effects on soil biota: A review. Soil Biology and Biochemistry (43): 1812-1836. Doi:10.1016/j.soilbio.2011.04.022.

Liang, B., J. Lehmann, D. Solomon, J. Kinyangi, J. Grossman, B. O'Neill, J. O. Skjemstad, J. Thies, F. J. Luizão, J. Petersen and E. G. Neves. 2006. Black carbon increases cation exchange capacity in soils. Soil Science Society of America Journal 70(5): 1719-1730. Doi: 10.2136/sssaj2005.0383.

Liu, X.-H., F.-P. Han and X.-C. Zhang. 2012. Effect of biochar on soil aggregates in the Loess Plateau: Results from incubation experiments. International Journal of Agriculture \& Biology 14:975-979. Doi:10.1016/j.geoderma.2020.114323.

Nunes D., Y., E. Santos S., H. S. Cardoso C., L. C. Acevedo M., E. S. Penido, C. Bastos A., O. M. Marques T. and A. R. Fernandes. 2019. Biochar produced from Amazonian agro-industrial wastes: properties and adsorbent potential of $\mathrm{Cd} 2+$ and Cu2+. Biochar 1(4): Doi: 10.1007/s42773-019-00031-4. 
Pérez G., G. 2015. Biocarbón (Biochar) de residuos forestales y agrícolas como alternativa para capturar carbono. Tesis de doctorado. Campus Montecillo, Edafología. Colegio de Postgraduados. Institución de Enseñanza e Investigación en Ciencias Agrícolas. Colegio de Postgraduados. Montecillo, Texcoco, Edo. de Méx., México. 103 p.

Rojas P., T. 2017. Uso del biochar para mejorar la calidad de plantas de café (Coffea arabica). Tesis de maestría. Campus Montecillo, Edafología. Colegio de Postgraduados. Institución de Enseñanza e Investigación en Ciencias Agrícolas. Colegio de Postgraduados. Montecillo, Texcoco, Edo. de Méx., México. 82 p.

Sánchez, S., M. Hernández y F. Ruz. 2011. Alternativas de manejo de la fertilidad del suelo en ecosistemas agropecuarios. Pastos y Forrajes 34(4): 375-392. http://scielo.sld.cu/scielo.pHp?script=sci_arttext\&pid=S0864-03942011000400001 (4 de junio 2020).

Secretaría de Medio Ambiente y Recursos Naturales (Semarnat). 2002. Norma oficial mexicana, NOM-021-RECNAT-2000, que establece las especificaciones de fertilidad, salinidad y clasificación de suelos. Estudios, muestreo y análisis. Diario Oficial de la Federación. 31 de diciembre de 2002. México, D.F, México. http://biblioteca.semarnat.gob.mx/janium/Documentos/Ciga/libros2009/DO2280n.pdf (1 de junio 2020).

Secretaría de Medio Ambiente y Recursos Naturales- Colegio de Postgraduados (Semarnat-Colpos). 2003. Evaluación de la degradación del suelo causada por el hombre en la República Mexicana, escala 1:250 000. Memoria Nacional 2001-2002. México, D.F., México. s/p.

Sitún, M. 2005. Investigación agrícola. Segunda Edición. Editorial ENCA. Bárcena, Villa Nueva, Guatemala. $137 \mathrm{p}$ 
Srinivasarao, C., K. A. Gopinath, G. Venkatesh, A. K. Dubey, H. Wakudkar, T. J. Purakayastha, H. Pathak, P. Jha, B. L. Lakaria, D. J. Rajkhowa, S. Mandal, S. Jeyaraman, B. Venkateswarlu and A. K. Sikka. 2013. Use of biochar for soil health management and greenhouse gas mitigation in India: Potential and constraints. Central Research Institute for Dryland Agriculture. Hyderabad, Andhra Pradesh, India. $51 \mathrm{p}$.

Steiner, C. 2010. Biochar in agriculture and forestry applications in: Biochar from Agricultural and Forestry Residues - A complimentary use of "waste" biomass. In: Levine, J. (ed.). Assessment of biochars benefits for the USA. United States Biochar Initiative. Center for Energy and Environmental Security. Boulder, CO, USA. 85 p.

Uchimiya, M., I. M. Lima, K. T. Klasson, S. Chang, L. H. Wartelle and J. E. Rodgers. 2010. Immobilization of heavy metal ions (CU-II, CdII, Ni-II, and Pb-II) by broiler litter-derived biochars in water and soil. Journal of Agricultural Food Chemistry 58 (9):5538-5544. Doi: 10.1021/jf9044217.

Una Norma Española-Norma Europea (UNE-EN). 2010. Biocombustibles sólidos. Determinación del contenido de humedad. Método de secado en estufa. Parte 2: Humedad total. Método simplificado. https://www.une.org/encuentra-tunorma/busca-tu-norma/norma?c=N0045727 (20 de noviembre de 2019).

Waters D., L. Van Zwieten, B. P. Singh, A. Downie, A. L. Cowie and J. Lehmann. 2011. Biochar in Soil for Climate Change Mitigation and Adaptation. In: Singh, B., A. Cowie and K. Chan (eds.). Soil Health and Climate Change. Soil Biology. Vol 29. Springer, Berlin, Heidelberg. Doi: 10.1007/978-3-642-20256-8_15. 
Yao, Y., B. Gao, J. Chen and L. Yang. 2013. Engineered biochar reclaiming pHospHate from aqueous solutions: Mechanisms and potential application as a slowrelease fertilizer. Environmental Science and Technology 47(15): 8700-8708. Doi: $10.1021 /$ es4012977.

Todos los textos publicados por la Revista Mexicana de Ciencias Forestales -sin excepciónse distribuyen amparados bajo la licencia Creative Commons 4.0 Atribución-No Comercial (CC BY-NC 4.0 Internacional), que permite a terceros utilizar lo publicado siempre que mencionen la autoría del trabajo y a la primera publicación en esta revista. 